\title{
Rate-Distortion Theory for Affine (Global) Motion Compensation in Video Coding
}

Holger Meuel

Institut für Informationsverarbeitung Leibniz Universität Hannover, Germany

Presentation of the ICIP 2018 paper at the SVCP, July $4^{\text {th }}-6^{\text {th }}, 2018$

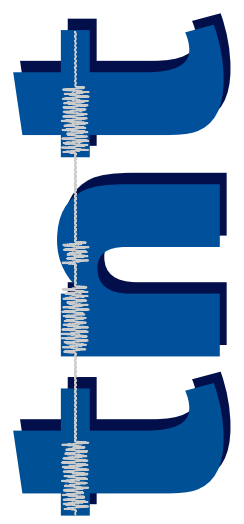




\section{Motivation}

- Motion compensated prediction (MCP) as one key element in hybrid video coding

- High dependence between accuracy of motion estimation (ME) and prediction error (PE)

- Inaccurate displacement estimation

$\Rightarrow$ High prediction error

$\Rightarrow$ High entropy

$\Rightarrow$ High bit rate

\section{Goal:}

Model the prediction error bit rate as a function of the displacement estimation error for an affine motion model

Original aerial frame (top) 


\section{Overview $^{2}$}

- Model of power spectral density (P.S.D.) of signal

- Model of probability density function (p.d.f.) of displacement estimation error

- Derivation of P.S.D. of displacement estimation error $S_{e e}(\Lambda)$

- Application of rate-distortion theory $\Rightarrow$ bit rate

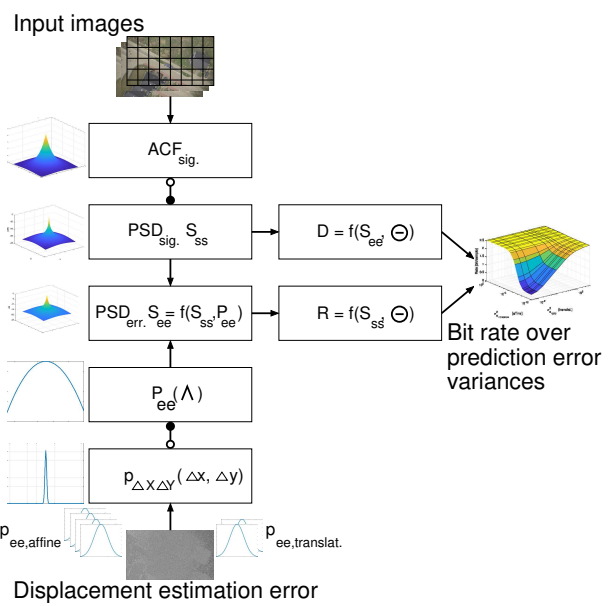

${ }^{2}$ Modeling based on B. Girod, "The Efficiency of Motion-Compensating Prediction for Hybrid Coding of Video Sequences," in IEEE Journal on Selected Areas in Communicat., vol. 5, no. 7, pp. 1140-1154, 1987 


\section{Outline}

Efficiency Analysis of Affine Motion Compensated Prediction

Model of the Probability Density Function (p. d. f.)

Model of Power Spectral Densities (P. S. D.s)

Rate-Distortion Analysis

Simulations

Conclusion 


\section{Outline}

Efficiency Analysis of Affine Motion Compensated Prediction

Model of the Probability Density Function (p. d. f.)

Model of Power Spectral Densities (P. S. D.s)

Rate-Distortion Analysis 


\section{Affine Motion Model and Error Model}

$$
\begin{aligned}
& x=a_{11} \cdot x^{\prime}+a_{12} \cdot y^{\prime}+a_{13} \\
& y=a_{21} \cdot x^{\prime}+a_{22} \cdot y^{\prime}+a_{23}
\end{aligned}
$$

- $a_{13}$ and $a_{23}$ translational parameters

- $a_{11,12,21,22}$ "purely affine" parameters (rotation, scaling and shearing)

- Perturbation (indicated by $\hat{\imath}$ ) by error terms $e_{i j}, i=\{1,2\}, j=\{1,2,3\}$ caused by inaccurate estimation

$$
\begin{aligned}
& \Delta x=\hat{x}-x=\underbrace{\left(\hat{a}_{11}-a_{11}\right)}_{e_{11}} \cdot x^{\prime}+\underbrace{\left(\hat{a}_{12}-a_{12}\right)}_{e_{12}} \cdot y^{\prime}+\underbrace{\left(\hat{a}_{13}-a_{13}\right)}_{e_{13}} \\
& \Delta x=e_{11} \cdot x^{\prime}+e_{12} \cdot y^{\prime}+e_{13} \\
& \Delta y=e_{21} \cdot x^{\prime}+e_{22} \cdot y^{\prime}+e_{23}
\end{aligned}
$$




\section{Probability Density Function (p.d.f.) of the Displacement}

\section{Estimation Error}

Assumption: $e_{i j}$ zero-mean Gaussian distributed with p.d.f.:

$$
\begin{aligned}
& p\left(e_{i j}\right)=\frac{1}{\sqrt{2 \pi \sigma_{e_{i j}}^{2}}} \cdot \exp \left(-\frac{e_{i j}^{2}}{2 \sigma_{e_{i j}}^{2}}\right) \\
& \text { with } i=\{1,2\} \text { and } j=\{1,2,3\}
\end{aligned}
$$

Joint p.d.f. for independent $e_{i j}$ :

$$
p_{E_{11}, \ldots, E_{23}}\left(e_{11}, \ldots, e_{23}\right)=p\left(e_{11}\right) \cdot \ldots \cdot p\left(e_{23}\right)
$$




\section{Probability Density Functions Conversion}

- Given now: joint p.d.f. $p_{E_{11}, \ldots, E_{23}}\left(e_{11}, \ldots, e_{23}\right)$

- Wanted: p.d.f. $p_{\Delta X, \Delta Y}(\Delta x, \Delta y)$ of displacement estimation errors $\Delta x, \Delta y$

With transformation theorem for p.d.f.s:

$$
\begin{aligned}
& p_{\Delta X, \Delta Y}(\Delta x, \Delta y)=\int_{\mathbb{R}^{6}} p_{E_{11}, \ldots, E_{23}}\left(e_{11}, \ldots, e_{23}\right) \\
& \cdot \delta\left(\Delta x-\left(x^{\prime} e_{11}+y^{\prime} e_{12}+e_{13}\right)\right) \\
& \cdot \delta\left(\Delta y-\left(x^{\prime} e_{21}+y^{\prime} e_{22}+e_{23}\right)\right) d e_{11} \ldots d e_{23}
\end{aligned}
$$




\section{Probability Density Function of the Displacement}

\section{Estimation Error}

$$
\begin{gathered}
p_{\Delta X, \Delta Y}(\Delta x, \Delta y)=\frac{1}{2 \pi \sigma_{\Delta x} \sigma_{\Delta y}} \cdot \exp \left(-\frac{\Delta x^{2}}{2 \sigma_{\Delta x}^{2}}\right) \cdot \exp \left(-\frac{\Delta y^{2}}{2 \sigma_{\Delta y}^{2}}\right) \\
\text { with } \sigma_{\Delta x}^{2}=\sigma_{e_{11}}^{2} x^{\prime 2}+\sigma_{e_{12}}^{2} y^{\prime 2}+\sigma_{e_{13}}^{2} \\
\text { and } \sigma_{\Delta y}^{2}=\sigma_{e_{21}}^{2} x^{\prime 2}+\sigma_{e_{22}}^{2} y^{\prime 2}+\sigma_{e_{23}}^{2}
\end{gathered}
$$

Variances $\sigma_{\Delta x}^{2}$ and $\sigma_{\Delta y}^{2}$ depend on locations $x^{\prime}, y^{\prime}$ ! 


\section{Location Dependent Variance $\sigma_{\Delta x}^{2}$ of Gaussian Distributed Displacement Estimation Error p.d.f.s for a Full HD Image}

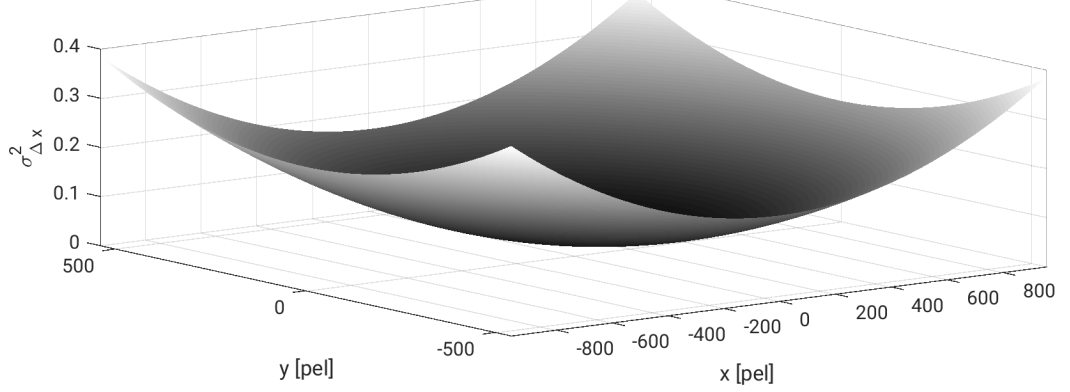

$\sigma_{e_{11}}^{2}=2.3 e-7, \sigma_{e_{12}}^{2}=4.6 e-7\left(\right.$ measured from $\left.\operatorname{TAVT}^{3}\right)$ and $\sigma_{e_{13}}^{2}=0.04\left(\right.$ like Girod $\left.{ }^{2}\right)$

${ }^{3}$ TNT Aerial Video Testset (TAVT), Institut für Informationsverarbeitung (TNT), Leibniz Universität Hannover, 2014, online: https://www.tnt.uni-hannover.de/project/TAVT/

${ }^{2}$ B. Girod, "The Efficiency of Motion-Compensating Prediction for Hybrid Coding of Video Sequences," in IEEE Journal on Selected Areas in Communications, vol. 5, no. 7, pp. 1140-1154, Aug 1987 


\section{Outline}

Efficiency Analysis of Affine Motion Compensated Prediction

Model of Power Spectral Densities (P.S. D.s)

Simulations

Conclusion 


\section{Signal and Error Power Spectral Density Functions}

- Assumption of isotropic autocorrelation function ${ }^{4}$ :

$$
\begin{aligned}
R_{s s}(\Delta x, \Delta y) & =E[s(x, y) \cdot s(x-\Delta x, y-\Delta y)] \\
& :=\exp \left(-\alpha \sqrt{\Delta x^{2}+\Delta y^{2}}\right)
\end{aligned}
$$

- Determination of power spectral density of the video signal (Wiener-Khinchin theorem):

$$
S_{s S}(\Lambda)=\mathcal{F}\left(R_{S S}(\Delta x, \Delta y)\right)
$$

- Power spectral density of displacement estimation error ${ }^{2}$ :

$$
S_{e e}(\Lambda)=2 S_{s S}(\Lambda)[1-\operatorname{Re}(P(\Lambda)]+\Theta
$$

${ }^{2}$ B. Girod, "The Efficiency of Motion-Compensating Prediction for Hybrid Coding of Video Sequences," in IEEE Journal on Selected Areas in Communicat., vol. 5, no. 7, pp. 1140-1154, Aug 1987 ${ }^{4} \mathrm{~J}$. O'Neal and T. Natarajan, "Coding Isotropic Images", IEEE Transact. on Inform. Theory, vol. 23, no. 6, pp. 697-707, Nov. 1977 


\section{Outline}

Efficiency Analysis of Affine Motion Compensated Prediction

Model of Power Spectral Densities (P. S. D.s)

Rate-Distortion Analysis

\section{Simulations}

Conclusion 


\section{Rate-Distortion Theory ${ }^{5}$}

$$
\begin{gathered}
D=\frac{1}{4 \pi^{2}} \iint_{\Lambda} \min \left[\Theta, S_{s s}(\Lambda)\right] \mathrm{d} \Lambda \\
R(D)=\frac{1}{8 \pi^{2}} \iint \log _{2}\left[\frac{S_{e e}(\Lambda)}{\Theta}\right] \mathrm{d} \Lambda \text { bit } \\
\wedge:\left(S_{s s}(\Lambda)>\Theta\right. \\
\text { and } \left.S_{e e}(\Lambda)>\Theta\right)
\end{gathered}
$$

with $\Theta$ being a parameter that generates the function $R(D)$ by taking on all positive real values

5 based on Toby Berger, "Rate Distortion Theory: A Mathematical Basis for Data Compression", Prentice-Hall electrical eng. series, Prentice-Hall, 1971 


\section{Outline}

\section{Efficiency Analysis of Affine Motion Compensated Prediction Model of the Probability Density Function (p. d. f.) Model of Power Spectral Densities (P. S. D.s) Rate-Distortion Analysis}

Simulations

\section{Conclusion}




\section{Pixel Correlations}

\begin{tabular}{|c|c|c|}
\hline Sequence & Corr. $\rho_{x}$ & Corr. $\rho_{y}$ \\
\hline Values from Girod & 0.928 & 0.934 \\
\hline 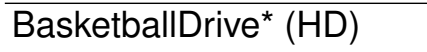 & 0.9782 & 0.9488 \\
\hline BQTerrace* (HD) & 0.9680 & 0.9659 \\
\hline Cactus* $^{*}(\mathrm{HD})$ & 0.9741 & 0.9812 \\
\hline Kimono* (HD) & 0.9883 & 0.9900 \\
\hline ParkScene* $(\mathrm{HD})$ & 0.9634 & 0.9518 \\
\hline Mean of HD sequences* & 0.9744 & 0.9677 \\
\hline
\end{tabular}

Measured horizontal and vertical correlations between adjacent pixels for typical test sequences ( ${ }^{*}: 100$ frames each). 


\section{Variances of Affine Transformation Parameters}

\begin{tabular}{|c|c|c|c|c|c|c|}
\hline & $\sigma_{e_{11}}^{2}$ & $\sigma_{e_{12}}^{2}$ & $\sigma_{e_{21}}^{2}$ & $\sigma_{e_{22}}^{2}$ & $\begin{array}{c}\text { mean } \\
\left(\sigma_{e_{11}}^{2}, \sigma_{e_{22}}^{2}\right)\end{array}$ & $\begin{array}{l}\text { mean } \\
\left(\sigma_{e_{12}}^{2}, \sigma_{e_{21}}^{2}\right)\end{array}$ \\
\hline $350 \mathrm{~m}$ seq. & $2.03 e-7$ & $6.03 e-7$ & $6.59 \mathrm{e}-7$ & $2.24 \mathrm{e}-7$ & $2.13 e-7$ & $6.31 e-7$ \\
\hline $500 m$ seq. & $1.94 \mathrm{e}-7$ & $5.09 e-7$ & $3.63 e-7$ & $1.94 \mathrm{e}-7$ & $1.94 e-7$ & $4.35 e-7$ \\
\hline $1000 \mathrm{~m}$ seq. & $1.74 \mathrm{e}-7$ & $4.05 e-7$ & $4.13 e-7$ & $2.12 e-7$ & $1.93 e-7$ & $4.09 e-7$ \\
\hline $1500 \mathrm{~m}$ seq. & $3.19 \mathrm{e}-7$ & $3.80 e-7$ & $3.69 e-7$ & $3.46 e-7$ & $3.33 e-7$ & $3.75 e-7$ \\
\hline Mean & $2.23 e-7$ & $4.74 e-7$ & $4.51 e-7$ & $2.44 e-7$ & $2.33 e-7$ & $4.63 e-7$ \\
\hline
\end{tabular}

Measured variances $\sigma_{e_{i j}}^{2}$ of affine transformation parameters of aerial videos from the TAVT data set ${ }^{3}$.

Note: $\sigma_{e_{11}}^{2}$ and $\sigma_{e_{22}}^{2}$ as well as $\sigma_{e_{12}}^{2}$ and $\sigma_{e_{21}}^{2}$ are pairwise similar.

${ }^{3}$ TNT Aerial Video Testset (TAVT), Institut für Informationsverarbeitung (TNT), Leibniz Universität Hannover, 2014, online: https://www.tnt.uni-hannover.de/project/TAVT/ 


\section{Simulated Location Dependent Bit Rate}

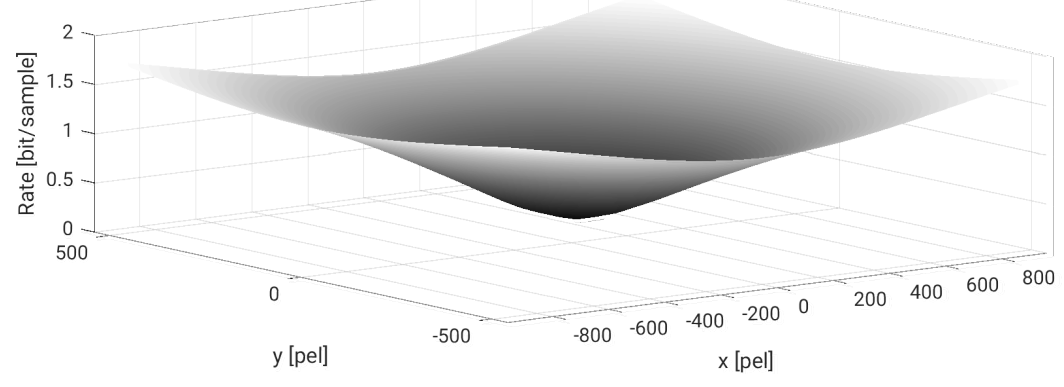

Bit rate

Simulation for Gaussian distributed displacement estimation error p.d.f.s for full $\mathrm{HD}$ image and variances $\sigma_{e_{11}}^{2}=\sigma_{e_{22}}^{2}=2.3 \mathrm{e}-7, \sigma_{e_{12}}^{2}=\sigma_{e_{21}}^{2}=4.6 \mathrm{e}-7$. 


\section{Minimum Required Bit Rate for Prediction Error Coding}

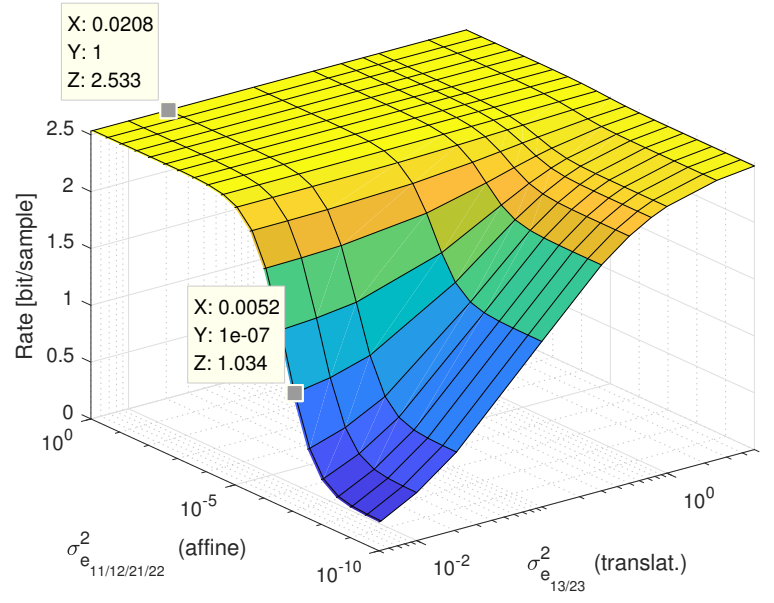

Distortion $\mathrm{SNR}=30 \mathrm{~dB}, \sigma_{e_{11}}^{2}=\sigma_{e_{12}}^{2}=\sigma_{e_{21}}^{2}=\sigma_{e_{22}}^{2}$ and $\sigma_{e_{13}}^{2}=\sigma_{e_{23}}^{2}$, full HD resolution. Datatips indicate isolines for translational quarter- $(0.0052)$ and half-pel resolution. 


\section{Outline}

\section{Efficiency Analysis of Affine Motion Compensated Prediction Model of the Probability Density Function (p. d. f.)

\section{Simulations}

Conclusion 


\section{Summary: RD Theory for Affine MCP in Video Coding}

Model for affine motion compensation in video coding:

- Model the displacement estimation error as a function of the motion estimation accuracy:

- Model the p.d.f. of displacement estimation error $p_{\Delta X, \Delta Y}(\Delta x, \Delta y)$

- Model the P.S.D. of video signal $S_{S S}$

- Derivation of power spectral density of displacement estimation error $S_{e e}$

- Application of rate-distortion function

$\Rightarrow$ Model for minimum required bit rate for prediction error coding

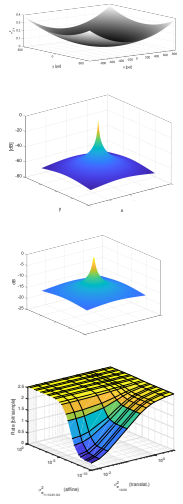

